\title{
Estrategia internacional, innovación y rendimiento: conglomerados estratégicos en pymes catalanas
}

\author{
International strategy, innovation and performance: \\ strategic clusters in Catalan SMEs
}

\section{Estratégia internacional, inovação e desempenho: os clusters estratégicos em pymes catalá}

\author{
M.B.A. Jorge Pinilla Jara* \\ Ph. D. Alex Criado**
}

\section{RESUMEN}

Este documento identifica cuatro tipos estratégicos de pymes internacionalizadas catalanas. Además, se realiza un análisis teórico de orientaciones y comportamientos estratégicos, seguido de una investigación empírica con una muestra de pymes catalanas. De los hallazgos empíricos, hay un grupo orientado al crecimiento de las empresas, otro orientado al cliente, un clúster orientado hacia el interior, y otro que carece de orientación estratégica. Por último, se confirma el papel positivo y significativo de los tipos estratégicos en las pymes, tradicionalmente estudiado solo en las multinacionales.

Palabras clave: orientaciones estratégicas, innovación, internacionalización de pymes.

\begin{abstract}
This paper identifies four strategic types of internationalized SMEs Catalan. It also carries out a theoretical analysis of strategic orientations and behaviors, followed by an empirical research with a sample of Catalan SMEs. Empirical findings suggest that there are a growth-oriented group of firms, another customer-oriented group, a inward-oriented cluster, and a further group of firms that lacks strategic orientation. Finally, we confirm the positive and significant role of strategic types in SMEs traditionally studied in multinationals.
\end{abstract}

Keywords: strategic orientations, innovation, internationalization of SMEs.

\section{RESUMO}

Este documento identifica quatro tipos estratégicos de pymes internacionalizadas catalâs. Além disso, realiza-se uma análise teórica das orientaçôes e comportamentos estratégicos, seguido por uma pesquisa empírica com uma amostra de pymes catalâs. Dos resultados empíricos, há um grupo orientado ao

* Chileno-Español, Ingeniero Comercial, M. B .A., M. Phil., Doctorando, Facultad de Economía y Empresa, Universidad Autónoma de Barcelona, Barcelona, España. Correspondencia con el autor: jorgealejandro.pinilla@uab.es.

** Español, Lic. Económicas, Ph. D., Profesor titular, Facultad de Economía y Empresa, Universidad Autónoma de Barcelona, Barcelona, España. Correspondencia con el autor: alex.rialp@ uab.es. 
Estrategia internacional, innovación y rendimiento: conglomerados estratégicos en pymes catalanas / Pinilla y Rialp

crescimento das empresas, outro orientado ao cliente, um cluster orientado ao interior e outro que carece de orientação estratégica. Finalmente, se confirma o papel positivo e significativo dos tipos estratégicos nas pymes, tradicionalmente estudado apenas nas multinacionais.

Palavras-chave: orientaçôes estratégicas, inovação, internacionalização das pymes.

\section{Introducción}

Existe un vacío de investigación sobre la relación entre estrategia e internacionalización. En este sentido, Melin (1992) no sólo hace hincapié en esto sino que también pone de relieve la naturaleza determinista y estática de la mayoría de las contribuciones. La investigación sobre negocios internacionales es un tema que ha sido descuidado y, específicamente, la investigación sobre posibles tipologías estratégicas alternativas es dispersa al igual que ocurre con la relación entre la orientación estratégica, la internacionalización y el rendimiento general de la empresa. Además, la gran mayoría de los artículos académicos revisados centran su atención en las grandes empresas multinacionales, mientras que la estrategia internacional de las pequeñas y medianas empresas (pymes) sigue siendo un campo de investigación en gran medida sin abordar (Bell et al., 2004).

En cuanto a la estrategia internacional de las pequeńas y medianas empresas -consideradas el motor de crecimiento económico y empleo- (Yongho et al., 2012; Henderson y Weiler, 2010), sigue siendo un campo de investigación de actualidad (Fernández y Alegre, 2015; Golovko y Valentini, 2011; Bell et al., 2004). Y en la investigación de negocios internacionales, el análisis de las orientaciones estratégicas diferenciadas de las Pymes en los mercados internacionales aún no ha sido suficientemente estudiado (Hagen et al., 2012), a pesar de que la relación entre las orientaciones estratégicas diferenciadas, los comportamientos estratégicos relacionados y el desempeño internacional, son temas relevantes para empresarios, gerentes y responsables políticos (ibídem).

La falta de investigación de las pymes a nivel de estrategia de negocio podría explicarse en parte por el hecho de que el comportamiento de las pymes se considera esencialmente no planificado y reactivo (Bilkey y Tesar, 1977). Sin embargo, algunos investigadores como Bell et al. (2004) señalan que la ausencia de una estrategia explícita y formal no significa una falta de visión estratégica.

La innovación e internacionalización desempeñan un papel preponderante en un entorno empresarial competitivo como el de las pymes, ya que son factores claves en el desempeño de la empresa y mutuamente dependientes (Denicolai et al., 2015; Añon y 
Driffield, 2011), además son considerados los factores más relevantes en el éxito de los negocios (Zahra et al., 2000; Vila y Kuster, 2007) y son reconocidas como importantes fuentes de crecimiento de la empresa (Prashantham, 2008).

Las investigaciones sobre tipologías estratégicas de empresas, orientación estratégica, internacionalización y rendimiento están dispersas. En tal sentido, esta contribución se centra en encontrar conglomerados estratégicos de pymes en función de las orientaciones estratégicas empresariales y relacionarlas con el tamaño de la empresa, sector económico, innovación e internacionalización, estos dos últimos como ejes de la investigación. Con el análisis empírico se pretende hallar tipologías de pequeñas y medianas empresas catalanas a partir de estrategias empresariales con el fin de analizar en qué medida estos conglomerados están relacionados con el desempeño de la pyme.

El documento está estructurado de la siguiente manera. En primer lugar, se realiza una revisión de la literatura de la investigación existente en las principales dimensiones del constructo estrategia, en particular, la dimensión de la orientación estratégica y sus vínculos con las estrategias competitivas y funcionales. En segundo lugar, se revisa el impacto de la orientación estratégica y tipos estratégicos sobre el desempeño. Posteriormente se presenta la metodología de investigación y se discuten los principales resultados. Finalmente, el documento termina con una discusión de las implicaciones del estudio para los profesionales e investigadores, sus limitaciones y la identificación de las oportunidades futuras de investigación.

\section{Constructos de orientaciones estratégicas}

En este trabajo se plantea la idea de que las orientaciones estratégicas en las pymes son diferenciadas, dando lugar también a comportamientos estratégicos diferenciados, determinando así rendimientos internacionales diferenciados. Los constructos se han definido teniendo presente toda la investigación sobre orientaciones estratégicas (OE) que existe. Los estudios sobre OE surgen de distintas corrientes de investigación, a saber, dirección estratégica, marketing estratégico y el emprendimiento internacional. $\mathrm{La} \mathrm{OE}$ son procesos, prácticas y toma de decisiones que conducen al crecimiento de una empresa (Lumpkin y Dess, 1996), y es considerada una parte importante de la cultura organizativa (Hunt y Morgan, 1995).

Existen diferentes enfoques en el estudio de las estrategias empresariales, como las de Ansoff (penetración de mercados. desarrollo de producto, desarrollo de mercados y diversificación), las de Porter (liderazgo en costos, diferenciación y de enfoque), las RBV de Barney (capacidades de la empresa y cómo los recursos permiten aplicar y crear 
Estrategia internacional, innovación y rendimiento: conglomerados estratégicos en pymes catalanas / Pinilla y Rialp

una ventaja competitiva), etc. Están más bien orientadas para las grandes empresas y no se han utilizado en la misma magnitud ni para las pymes ni para comprender la orientación estratégica internacional de las mismas.

Una de las principales contribuciones sobre $\mathrm{OE}$ diferenciada la proporcionan Miles y Snow (1978), quienes propusieron cuatro tipologías estratégicas basadas en la relación de empresas y su entorno, y que se adapta a los niveles diferenciados de proactividad estratégica, a saber: reactores (que carecen de una estrategia consistente), defensores (que adoptan una visión estratégica conservadora), exploradores (que ponen énfasis en la innovación y el cambio y compiten mediante la exploración de nuevas oportunidades de mercado, tendencias y tecnologías emergentes) y analizadores (es una mezcla de explorador y defensor y están en el medio de estas dos tipologías). Tanto defensores como exploradores, son dos polos opuestos estratégicamente según este análisis.

Aunque la metodología de Miles y Snow está bien fundamentada, se limitan únicamente a la comparación intergrupo, impidiendo cualquier investigación de análisis intragrupo (Speed, 1993). Desde que Miles y Snow (1978) introdujeron sus cuatro tipologías estratégicas, han surgido diversos enfoques alternativos donde gran parte de los trabajos sobre orientaciones estratégicas diferenciadas se pueden encontrar en la literatura de marketing, como orientación al mercado, orientación al producto, orientación al cliente, orientación a la innovación, orientación relacional, orientación a los accionistas, etc. (Human y Naudé, 2009). Aunque parece concebible que una empresa pueda adoptar múltiples orientaciones estratégicas, la idea de la orientación al mercado (Kohli y Jaworski, 1990; Narver y Slater, 1990) parece haber atraído la mayor parte de la atención en la literatura de marketing, porque tienden a demostrar una relación positiva entre la orientación al mercado y el desempeńo de la empresa.

El constructo de orientación al mercado $(O M)$ se encuentra en el centro del marketing moderno. El concepto se remonta a Drucker (1954) quien argumentó que los clientes deben ser la base de una organización y la razón de su existencia. El desarrollo del concepto de orientación al mercado incluye dos grandes sub-dimensiones, la orientación al cliente y la orientación al competidor. Este constructo pone énfasis en la creación de valor para el cliente y en un desempeño superior para la empresa (Narver y Slater, 1990). La OM se define como una cultura que pone la máxima prioridad en la creación y mantenimiento de valor para el cliente (Kohli y Jaworski, 1990; Narver y Slater, 1990). La empresa orientada al mercado está bien posicionada para anticiparse a las necesidades cambiantes de sus clientes y responder a éstos a través de la introducción de productos y servicios innovadores (Slater y Narver, 1995). La $\mathrm{OM}$ se caracteriza por el grado en que las empresas adquieren, difunden y responden 
a la información obtenida de los clientes, de los canales y competidores (Jaworski y Kohli, 1993; Kohli y Jaworski, 1990; Kohli et al., 1993). La gran mayoría de los estudios sobre $\mathrm{OM}$ han indagado el efecto de ésta en el rendimiento del negocio, lo que demuestra su superioridad como orientación estratégica (Zhou et al., 2005). Sin embargo, algunos estudios muestran que otras orientaciones también pueden influir en la ventaja competitiva y el rendimiento de las empresas (Fritz, 1996; Noble et al., 2002). Una de las sub-dimensiones de la orientación al mercado es la orientación al cliente, utilizado como sinónimo de la $\mathrm{OM}$ y como una dimensión del constructo "orientación al mercado" (Narver y Slater, 1990), pero la OM es un concepto más amplio.

La orientación emprendedora $(O E)$ es un enfoque emprendedor para las prácticas y formas de la toma de decisiones (Wiklund y Shepherd, 2005). Las empresas se caracterizan por ser autónomas, agresivas hacia la competencia, proactivas, innovadoras y dispuestas a correr riesgos (Lumpkin y Dess, 1996). La OE es un constructo compuesto por tres dimensiones: la capacidad de innovación, asumir riesgos y proactividad (Wiklund y Shepherd, 2005). Estas características pueden ocurrir en diferentes combinaciones en función del tipo de oportunidad empresarial a que la empresa se enfrente (Lumpkin y Dess, 1996). La capacidad de innovación se refiere a la tendencia de una empresa para llevar a cabo y fomentar nuevas ideas (Lumpkin y Dess, 1996), de manera que la empresa pueda cumplir con las necesidades del cliente ( $\mathrm{Li}$ et al., 2008). La proactividad se refiere a ser el primero en introducir nuevos productos en los mercados o anticiparse a la demanda futura (Keh et al., 2007). La capacidad de la empresa de asumir riesgos, se hace con la esperanza de obtener una alta rentabilidad ( $\mathrm{Li}$ et al., 2008). Las empresas con una mayor OE tienen un buen know-how sobre marketing, prestan especial atención a la identificación de necesidades del cliente, a la planificación y al rendimiento a largo plazo (Smart y Conant, 1994).

El constructo de orientación al aprendizaje (OA) se desarrolla a partir del aprendizaje organizativo como el desarrollo de conocimientos que influyen en el comportamiento de los empleados (Huber, 1991) y es el resultado de un comportamiento organizacional proactivo (Baker y Sinkula, 1999; Hult et al., 2004). La OA se define como un conjunto de valores organizacionales que influyen en la empresa para crear y utilizar todo tipo de conocimientos (Sinkula et al., 1997), y tiene tres dimensiones: a) compromiso con el aprendizaje, entendido como el grado en que una organización valora y promueve el aprendizaje (Sinkula et al., 1997), considerado éste como una inversión a largo plazo (Calantone et al., 2002); b) capacidad para desaprender lo aprendido (desaprendizaje), cuando las organizaciones de manera proactiva cuestionan sus rutinas y creencias promoviendo el desaprendizaje (Baker y Sinkula, 1999), y 
Estrategia internacional, innovación y rendimiento: conglomerados estratégicos en pymes catalanas / Pinilla y Rialp

c) apertura y experimentación, cuando con el paso del tiempo los modelos mentales tradicionales se vuelven obsoletos ante los cambios suscitados en el entorno (Baker y Sinkula, 1999). Las empresas con una mayor OA exigen a los empleados cuestionar constantemente las normas de la organización (Sinkula, 1994; Sinkula et al., 1997). Las empresas orientadas al aprendizaje son capaces de predecir los cambios del entorno, del mercado, y realizar los cambios necesarios si se requiere (Calantone et al., 2002). La adopción de una OA permite un mejor desempeño de la organización, crear conocimientos, competencias y responder mejor a su entorno cambiante (Baker y Sinkula, 1999; Liu et al., 2002; Slater y Narver, 1995).

La orientación a la innovación (OI) es el factor clave para mejorar la capacidad de una empresa cuando utiliza con éxito nuevos sistemas, ideas, procesos o productos (Hult et al., 2004; Hurley y Hult, 1998) y se asocia con inversiones en liderazgo tecnológico y con productos de alta calidad (Fritz, 1996; Gatignon y Xuereb, 1997). La OI pone énfasis en la creatividad, nuevas ideas, adopción de tecnologías, recursos, habilidades y sistemas administrativos nuevos (Hurley y Hult, 1998). La OI ha demostrado tener consecuencias positivas en el rendimiento del negocio, ya que los clientes prefieren los productos y servicios de alta calidad e innovadores (Berthon et al., 2004; Simpson et al., 2006).

Por último, la orientación al producto $(O P)$, dentro de la literatura de marketing, tiene como finalidad la producción de productos y servicios disponibles y de bajo precio para tener una ventaja competitiva (Kotler, 2002; Noble et al., 2002), donde el precio es razonable para un producto de calidad (Zhou y Li, 2007). La OP tiene un impacto positivo en el rendimiento del negocio (Noble et al., 2002). Este enfoque ha sido aplicado con éxito en industrias, más allá de la configuración tradicional de fabricación (Kotler, 2002). La mayoría de las empresas que practican la OP pasan una parte de sus beneficios a los consumidores en forma de precios más bajos, otra parte es retenida por la empresa, que se traduce en un rendimiento financiero superior (Noble et al., 2002).

Según el análisis anterior, se puede afirmar que existe una estrecha relación entre OM y OI (Hurley y Hult, 1998). La OM y la OI están relacionadas, aunque reflejen distintas filosofías empresariales (Zheng et al., 2005). También hay una relación entre la OI y gestión del conocimiento (Amar y Juneja, 2008). Con el fin de estimular la innovación, capturar, compartir, transferir y crear conocimiento en una organización, la gestión de conocimiento debe ser la piedra angular según Liebowitz (2008). El conocimiento es reconocido como un antecedente fundamental para la innovación (Darroch y McNaughton, 2002). La gestión del conocimiento así como las prácticas de recursos humanos, pueden interactuar de diversas formas para mejorar los resul- 
tados de la empresa (Aragón et al., 2015). La base de todos los conocimientos, en particular el componente que trae la innovación, está en el conocimiento tácito, es decir, lo que tácitamente las personas conocen o saben (Polanyi, 1966).

A partir de la revisión anterior se destaca: a) que los constructos relacionados con orientaciones estratégicas están profundamente arraigados a las corrientes de investigación que las generaron, esto quiere decir, que los diferentes constructos sobre orientación estratégica claramente tienen algunos rasgos comunes, pero las diferencias entre ellos no son siempre claras. Algunos autores han puesto en relieve este tema analizando los efectos sinérgicos de los constructos (Baker y Sinkula, 2009; Han et al., 1998), b) muchos de estos constructos son dicotómicos, esto quiere decir que las empresas o bien tienen una orientación dada o no, donde en realidad, las empresas pueden adoptar una variedad de orientaciones estratégicas, y c) se la ha prestado poca atención a las Pymes en la orientación estratégica internacional.

\section{Innovación e internacionalización en pymes}

La innovación y la internacionalización son reconocidos como dos principales fuentes de crecimiento de una empresa (Prashantham, 2008; Vila y Kuster, 2007). La relación entre la capacidad de innovación y el desempeño de la exportaciones no es uniforme, sino más bien depende de la configuración organizativa de la empresa (Yi et al., 2013). 
Estrategia internacional, innovación y rendimiento: conglomerados estratégicos en pymes catalanas /

Pinilla y Rialp

\begin{tabular}{|c|c|c|c|c|c|c|c|}
\hline 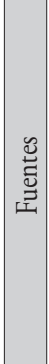 & 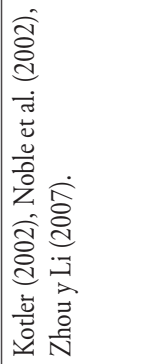 & 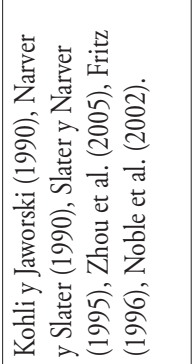 & 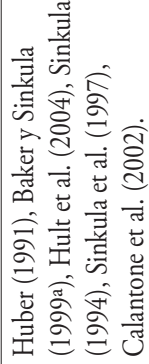 & 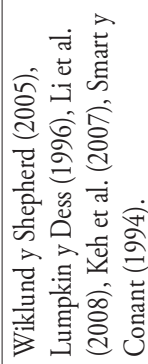 & 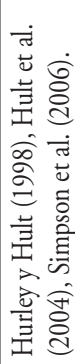 & 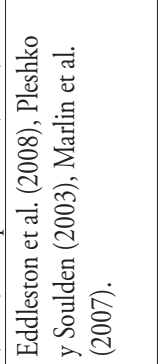 & 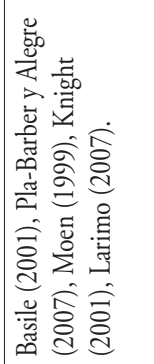 \\
\hline 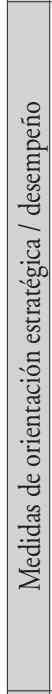 & 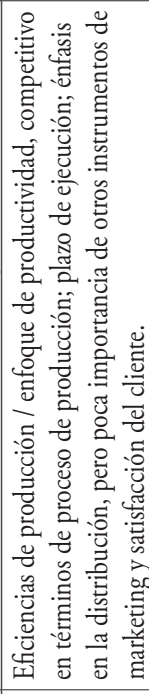 & 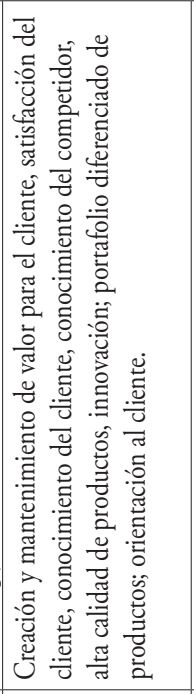 & 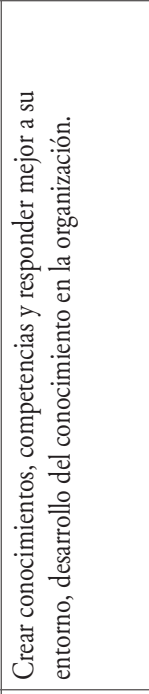 & 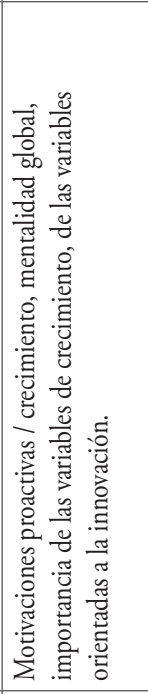 & 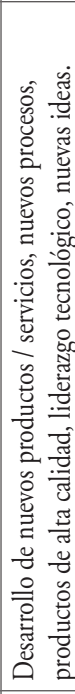 & 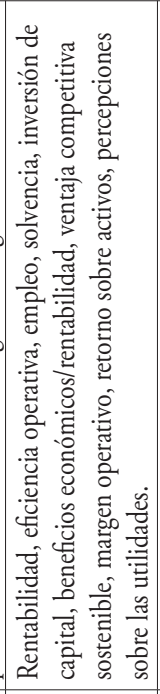 & 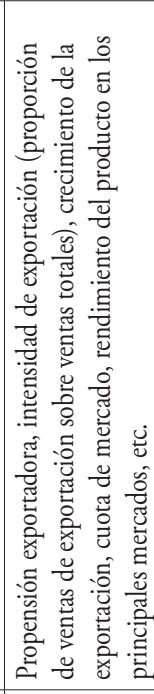 \\
\hline 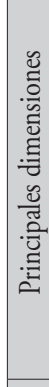 & 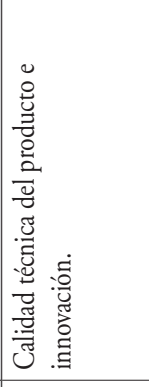 & 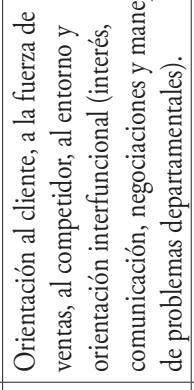 & 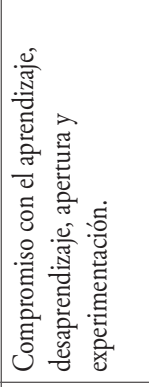 & 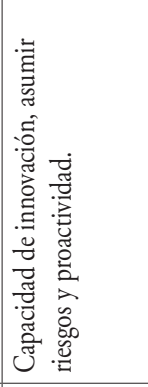 & 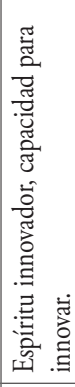 & 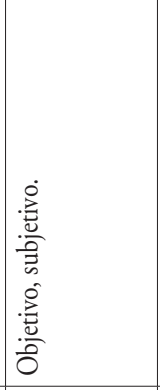 & 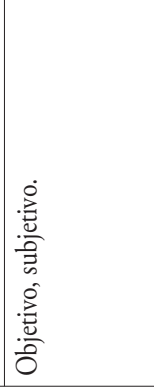 \\
\hline 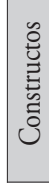 & 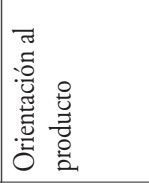 & 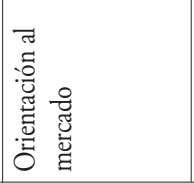 & 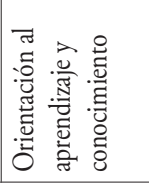 & 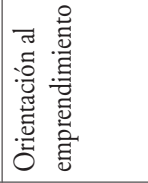 & 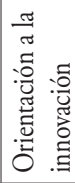 & 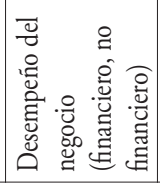 & 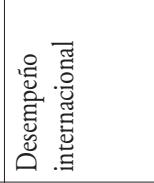 \\
\hline & 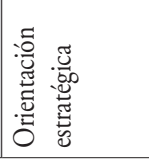 & & & & & 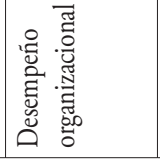 & \\
\hline
\end{tabular}


Según Ernst y Unctad (2005) la internacionalización fomenta la innovación, mientras que la innovación aumenta las oportunidades de internacionalización (Kotabe et al., 2007). La internacionalización tiende a enriquecer la capacidad de una empresa y mejora su rendimiento a través de la innovación (Kafouros et al., 2008). Para Cassiman y Golovko (2011) la decisión de una empresa de entrar en los mercados internacionales es en sí misma un proceso innovador, en cambio, para Schumpeter $(1934,1942)$ la innovación es determinante en el crecimiento económico y es una alternativa para crecer como empresa y mejorar el rendimiento organizativo. Por ende, la innovación está asociada con la creación y el uso del conocimiento en las empresas (Khilji et al., 2006; Palacios et al., 2009), lo que le permite obtener productos/servicios mejores que la competencia y una ventaja competitiva para competir en mercados locales e internacionales (Dibrell, et al., 2008; Madrid, et al., 2009).

La innovación ha demostrado tener una fuerte e influyente relación con el desempeño de una pyme (Verhees y Meulenberg, 2004) e induce a las empresas a aumentar sus actividades de exportación (Monreal-Pérez et al., 2012). Para Halilem (2014), la innovación favorece teóricamente la internacionalización. Yu y Si (2012) sostienen que las empresas que utilizan diferentes sistemas de innovación y participan activamente con programas de colaboración extranjera tienden a seguir una estrategia internacional. La innovación de productos tiende a fomentar las exportaciones (Oke et al., 2007; Ruzzier et al., 2006) y la combinación de innovación de procesos y productos, aumenta la propensión a exportar (Van Beveren y Vandenbussche, 2010). Existe pues una asociación entre innovación e internacionalización (Almeida y Fernandes, 2008; Andersson y Loof, 2011). Becker y Egger (2007) encontraron que la innovación era importante para exportar, lo mismo que Damijan et al. (2010).

En cuanto a la expansión internacional, la exportación es una estrategia de internacionalización para las pymes (Leonidou y Katsikeas, 1996). La internacionalización se produce cuando la empresa aumenta sus ventas o realiza otras actividades de negocio en mercados internacionales (Knight y Liesch, 2002), además de fomentar y mejorar el rendimiento de la innovación (Kafouros et al., 2008). También, la internacionalización ayuda a las empresas a ser más innovadoras (Damijan et al., 2010). Para Cassiman y Golovko (2011) la internacionalización crea oportunidades para desarrollar productos innovadores. Pero las empresas no están exentas de dificultades (Cuervo-Cazurra et al., 2007), ya que no hay certeza sobre el retorno de los recursos invertidos. Para ser competitivos en los mercados internacionales, Nieto y Santamaría (2010) encontraron que la intensidad de las exportaciones influye positivamente en los resultados de la innovación. Un intercambio de información relativamente abierto con los socios extranjeros estimula las actividades innovadoras en una empresa (Becker y Dietz, 2004; Furman et al., 2002; Hessels, 2007; Lundvall, 2007; Tether, 2002). 
Estrategia internacional, innovación y rendimiento: conglomerados estratégicos en pymes catalanas / Pinilla y Rialp

Sin embargo, la literatura económica presenta algunas inconsistencias con la relación innovación e internacionalización (Schubert y Simar, 2010; Woerter y Roper, 2010; Damijan et al., 2010; Cassiman y Martínez-Ros, 2007). D’Angelo (2012) encontró que el uso de $\mathrm{I}+\mathrm{D}$ tiene un impacto positivo y significativo en la intensidad de la exportación, mientras que el gasto en I+D no lo tiene. Golovko y Valentini (2011) encontraron un efecto positivo de la actividad de innovación en las empresas en crecimiento que se dedican a la exportación, y viceversa. Algunos estudios cuantitativos encontraron que las Pymes que recibieron subvenciones públicas eran más propensas a participar en la innovación de productos y procesos (Roper et al., 2008), mientras que otros no (Nauwelaerts y Vijfeyken, 2013). El apoyo del gobierno es importante para la internacionalización de las Pymes (Brewer, 2009), en cambio Bannò et al. (2013) y Yannopoulos (2010) encontraron que el impacto del apoyo del gobierno en la internacionalización depende de la experiencia de internacionalización, las estrategias, los recursos dedicados al proceso de internacionalización y las capacidades. Van Beveren y Vandenbussche (2010) encontraron una relación positiva entre la innovación en productos y procesos y exportación. Por su parte, Becker y Egger (2007) encontraron una importancia dominante de la innovación en productos para la decisión de exportar, en cambio otros estudios encontraron una importancia dominante de la innovación en procesos para la exportación (Damijan et al., 2010).

\section{Relación entre $\mathrm{OE}$, comportamiento estratégico y rendimiento internacional}

Examinar únicamente el constructo orientación estratégica proporciona una comprensión incompleta de rendimiento de las pymes, porque mientras ésta se relaciona con la ventaja competitiva, poco se sabe acerca de cómo se desarrollan los activos para alcanzar un rendimiento (Morgan et al., 2009). Diferentes orientaciones estratégicas conllevan a que las empresas se anticipen y reaccionen frente a factores internos y externos de varias maneras y que esto afecte sus procesos de exploración y explotación de oportunidades. Homburg et al. (2004), en línea con Narver y Slater (1990), identifican una relación entre la orientación al mercado y diferenciación para las grandes empresas, y Pelham y Wilson (1996) agregan evidencia en el contexto de las pymes. Las estrategias de liderazgo en costes, destinadas a alcanzar ventajas de costes en general, se han relacionado con orientaciones más defensivas y conservadoras (Miles y Snow, 1978; Porter, 1980) y también pueden estar relacionadas con la orientación a la producción.

En cuanto a las estrategias funcionales, las empresas exploradoras, emprendedoras y orientadas al mercado se han caracterizado por poner énfasis en el desarrollo de nuevos productos y servicios. Por lo general, anuncian y promocionan su producción, y son 
capaces de cobrar precios superiores a la media, mostrando una orientación de crecimiento superior (Buzzel y Gale, 1987; Hambrick, 1983; McKee et al., 1989; Miles y Snow, 1978; Miles y Snow, 1986). Por el contrario, las empresas defensoras, los negocios orientados internamente y los negocios orientados al producto, podrían ser descritos como aquellos que valoran los procesos de $\mathrm{I}+\mathrm{D}$, dirigiendo su atención hacia la reducción de los costes de fabricación y distribución, y tienden a poner énfasis en su producto, ya sea en el precio o en la calidad, y le asignan poco valor a la orientación al cliente (Dess y Davis, 1984; Miles y Snow, 1986; Porter, 1980; Wright et al., 1995). Por lo tanto, se pueden encontrar diferentes orientaciones estratégicas que varían en función de cómo las empresas valoran las diferentes estrategias competitivas y funcionales facilitadas por sus diferentes ventajas competitivas.

\section{Hipótesis}

Ninguna orientación estratégica por sí sola conduce a un rendimiento superior en todas las situaciones (Miles y Snow, 1978; Noble et al., 2002). Sin embargo, hay una falta de estudios que vinculan tipos de estrategias u orientaciones estratégicas al rendimiento en un contexto internacional y esto es especialmente para los hallazgos relacionados con las pymes (Balabanis y Katsikeas, 2003; Caruana et al., 1998). Por lo tanto:

$H_{1}$ : Las estrategias u orientaciones estratégicas influyen en el rendimiento de una pyme.

Algunas investigaciones (Kirca et al. (2005) vinculan positivamente la orientación al mercado con el rendimiento de una empresa, los cuales han sido confirmados mediante la técnica de metaanálisis. Hay algunas investigaciones en la orientación al mercado en el contexto internacional (Armario et al., 2008; Cadogan et al., 2003; Kirca et al., 2009) que muestran resultados mixtos. Por lo tanto:

$H_{2}$ : Existe una relación positiva entre la orientación al mercado y el rendimiento de una pyme.

Se ha descrito la orientación emprendedora como un antecedente para el crecimiento y rendimiento en las empresas tanto en los mercados nacionales como extranjeros (Kuivalainen et al., 2007; Robertson y Chetty, 2000). En general, la investigación sobre rendimiento exportador marca el impacto positivo de una postura proactiva y apoyo de la dirección, sumado al compromiso en el desempeño de las exportaciones (Madsen, 1989). Por lo tanto:

$H_{3}$ : Existe una relación positiva entre la orientación al emprendimiento, el rendimiento de una pyme y la internacionalización, relación que se ve reforzada cuando existe un compromiso y una actitud proactiva por parte de la dirección. 
Estrategia internacional, innovación y rendimiento: conglomerados estratégicos en pymes catalanas / Pinilla y Rialp

La investigación respecto a la orientación de innovación, medida por el éxito de innovación, muestra una relación positiva con el rendimiento del negocio y la rentabilidad (Gatignon y Xuereb, 1997; Han et al., 1998) en el ámbito de las pyme.

$H_{\dot{4}}$ Existe una relación positiva entre la orientación a la innovación y el rendimiento de una pyme.

En la revisión de la literatura también se encontraron resultados mixtos en los estudios empíricos relacionados con las tipologías estratégicas de Miles y Snow. Los resultados varían de resultados insignificantes (Miles y Snow, 1978), a significativos, pero con relaciones débiles (Conant et al., 1990). Woodside et al. (1999) encontraron una fuerte relación al vincular tipos estratégicos de desempeño organizacional a través de las competencias de marketing distintivo.

Los resultados de los estudios mixtos en este campo de investigación han sido atribuidos a los diversos funcionamientos de los constructos, pero también al hecho de que las relaciones son complejas y que las relaciones individuales y directas podrían no ser adecuadas para modelar correctamente (Baker y Sinkula, 2009; Lumpkin y Dess, 1996). Esta investigación incluye variables estratégicas empresariales vinculadas a la innovación e internacionalización. Bahadir et al. (2009) argumentan que diferentes factores que influyen en la innovación pueden llevar a diferentes resultados. Ar y Baki (2011) hallaron que tanto la innovación en procesos como en productos tienen una asociación fuerte y positiva con el desempeño de una pyme y March-Chorda et al. (2002) demuestran el excesivo coste del proceso de innovación en una pyme y la incertidumbre de nuevos productos en el mercado. La gestión del conocimiento, por su parte, apoya a la innovación en las organizaciones (Snowden, 2003).

La investigación se centra en la definición de conglomerados estratégicos en las pymes y se incorpora la interacción internacionalización-innovación y el rendimiento para una mejor interpretación de los resultados; en tal sentido, las ventas totales son un buen indicador de rendimiento (Delmar et al., 2003). Se trata de un indicador consistente para las investigaciones sobre la innovación (Helfat, 2007) y la internacionalización (Sapienza et al., 2006).

\section{Datos y metodología}

En España, el 99,9\% del tejido empresarial está constituido por pymes; en Cataluña es el 99,86\% y en Europa las pymes representan el 99,8\% del total de empresas (Annual Report on European SMEs 2013/2014). 


\subsection{Datos}

La investigación se ha llevado a cabo con los datos de un cuestionario de autoevaluación estructurado con preguntas cerradas desarrolladas por un equipo de profesionales del Gobierno de Cataluña, quienes realizaron un pre-testeo localmente con el fin de mejorar la tasa de respuesta y participación de las empresas. Los datos son de corte transversal (cross-section), fueron recogidos entre 2009 y 2012 y corresponden a 272 pymes catalanas de diecisiete sectores económicos.

Se obtuvo una muestra representativa de las pymes innovadoras del tejido empresarial de Cataluńa (1550) gestionadas por la Agencia para la competitividad de la empresa (ACCIÓ), organismo público dependiente de la Generalitat, en la cual participaron directores, gerentes, socios y responsables de pymes catalanas de las áreas de innovación e internacionalización. La tasa de respuesta en el período de recogida de datos fue del $18 \%$, que se puede considerar aceptable (Harzing, 2000), pero también comparable a otras investigaciones similares (Frambach et al., 2003; Gatignon y Xuereb, 1997). No se encontraron diferencias significativas entre los encuestados. El cuestionario consta de 47 preguntas. La tabla 1 resume las dimensiones sugeridas por la literatura. Se utilizó una escala Likert de cinco puntos (donde $1=$ no es importante y $5=$ es muy importante) para medir las diversas dimensiones de las orientaciones estratégicas, estrategia competitiva / funcional, ventaja competitiva y el rendimiento internacional. Las 272 empresas pueden, por lo tanto, ser consideradas como representativas de la población de pequeńas y medianas empresas correspondientes a pymes catalanas de diecisiete sectores económicos (ver tabla 2).

Tabla 2. Perfil de las empresas entrevistadas.

\begin{tabular}{|c|c|c|c|c|c|}
\hline & $\begin{array}{c}\mathrm{N}^{\circ} \\
\text { empresas }\end{array}$ & $\begin{array}{c}\mathrm{N}^{\circ} \text { Trabajadores } \\
\overline{\mathrm{x}}\end{array}$ & $\begin{array}{c}\text { Fact. Anual (1) } \\
\overline{\mathbf{x}}\end{array}$ & $\begin{array}{c}\% \text { Export. s/fact. } \\
\overline{\mathbf{x}}\end{array}$ & $\begin{array}{c}\text { Muestra } \\
(272)\end{array}$ \\
\hline PYME internacionales & & & & & 157 \\
\hline PYME mercado nacional & & & & & 115 \\
\hline Empleados (promedio) & & & & & 43 \\
\hline $\begin{array}{l}\text { Sector } 1 \text { Alimentación y } \\
\text { bebidas }\end{array}$ & 14 & 58 & 10 & 24 & $5,15 \%$ \\
\hline $\begin{array}{l}\text { Sector } 2 \text { Construcción y } \\
\text { materiales }\end{array}$ & 11 & 66 & 20 & 17 & $4,04 \%$ \\
\hline Sector 3 Distribución & 6 & 25 & 6 & 16 & $2,21 \%$ \\
\hline $\begin{array}{l}\text { Sector } 4 \text { Educación y } \\
\text { formación }\end{array}$ & 3 & 13 & 1 & 7 & $1,10 \%$ \\
\hline $\begin{array}{l}\text { Sector } 5 \text { Equipos eléctricos } \\
\text { y electrónicos }\end{array}$ & 22 & 49 & 22 & 29 & $8,09 \%$ \\
\hline
\end{tabular}


Estrategia internacional, innovación y rendimiento: conglomerados estratégicos en pymes catalanas / Pinilla y Rialp

\begin{tabular}{|c|c|c|c|c|c|}
\hline & \begin{tabular}{c|}
$\mathrm{N}^{\circ}$ \\
empresas
\end{tabular} & $\begin{array}{c}\mathrm{N}^{\circ} \text { Trabajadores } \\
\overline{\mathrm{x}}\end{array}$ & $\begin{array}{c}\text { Fact. Anual (1) } \\
\qquad \overline{\mathbf{x}}\end{array}$ & $\begin{array}{c}\text { \% Export. s/fact. } \\
\overline{\mathbf{x}}\end{array}$ & $\begin{array}{c}\text { Muestra } \\
(272)\end{array}$ \\
\hline Sector 7 Industria química & 36 & 40 & 9 & 37 & $13,24 \%$ \\
\hline $\begin{array}{l}\text { Sector } 8 \text { Industria del } \\
\text { papel y artes gráficas }\end{array}$ & 3 & 15 & 3 & 21 & $1,10 \%$ \\
\hline $\begin{array}{l}\text { Sector } 9 \text { Logística y } \\
\text { transporte }\end{array}$ & 6 & 41 & 10 & 37 & $2,21 \%$ \\
\hline Sector 10 Medioambiente & 5 & 18 & 2 & 2 & $1,84 \%$ \\
\hline $\begin{array}{l}\text { Sector } 11 \text { Metalúrgica, } \\
\text { equipos metálicos }\end{array}$ & 58 & 45 & 8 & 28 & $21,32 \%$ \\
\hline $\begin{array}{l}\text { Sector } 12 \text { Restauración y } \\
\text { turismo }\end{array}$ & 1 & 8 & 0 & 0 & $0,37 \%$ \\
\hline $\begin{array}{l}\text { Sector } 13 \text { Servicios y } \\
\text { material sanitario }\end{array}$ & 10 & 15 & 1 & 16 & $3,68 \%$ \\
\hline $\begin{array}{l}\text { Sector } 14 \text { Textil y } \\
\text { confección }\end{array}$ & 9 & 82 & 12 & 38 & $3,31 \%$ \\
\hline $\begin{array}{l}\text { Sector } 15 \text { TIC y } \\
\text { telecomunicaciones }\end{array}$ & 42 & 43 & 5 & 15 & $15,44 \%$ \\
\hline Sector 16 Otras industrias & 19 & 45 & 9 & 22 & $6,99 \%$ \\
\hline Sector 17 Otros servicios & 20 & 19 & 2 & 11 & $7,35 \%$ \\
\hline
\end{tabular}

(1) en millones de euros.

Fuente: elaboración propia.

Se utilizaron preguntas relacionadas con las características y actitudes de gestión e internacionalización (17 variables) para describir la proactividad/reactividad y la toma de riesgos, similar a las variables desarrolladas por Wood y Robertson (1997) y Moen (1999). Las preguntas sobre gestión del conocimiento (talento, fuente de ideas en proyectos relacionados con la innovación) suman 7. Las preguntas relacionadas con la orientación estratégica (emprendimiento, innovación, producto, proceso, ventas y orientación al mercado) totalizan 18 variables. También fueron juzgadas reveladoras de la fuente de ventaja competitiva basada en el concepto presentado por Day y Wensley (1988). Las preguntas sobre estrategia funcional (marketing) incluyen 5 variables.

La tabla 3 muestra la distribución de empresas por tamaño, considerando la normativa europea (Comisión de la UE 2003/361/CE de 6 de mayo de 2003). 
Tabla 3. Distribución de empresas por tamaño.

\begin{tabular}{|l|c|}
\hline \multicolumn{1}{|c|}{ Tamaño } & $\mathrm{N}^{\circ}$ de empresas \\
\hline Empleados $(0-10)$ & 65 \\
\hline Empleados $(11-50)$ & 139 \\
\hline Empleados $(51-250)$ & 68 \\
\hline Total & 272 \\
\hline
\end{tabular}

Fuente: elaboración propia.

\subsection{Metodología}

Se ha utilizado el análisis de conglomerados, como un método analítico y estructurado, para detectar grupos estratégicos homogéneos. Es un método estadístico multivariante que permite clasificar las empresas en conglomerados con características similares. Es una técnica estadística utilizada en una variedad de disciplinas cuando el objetivo es la clasificación de sujetos. Esta técnica permite conocer las características, el grado de importancia y significación estadística de los conglomerados (Stock y Zacarías, 2011), y permite la inclusión de múltiples variables permitiendo descripciones potencialmente ricas (Ketchen y Shook, 1996). En un contexto internacional, Morrison y Roth (1992) utilizaron esta técnica para detectar las estrategias de negocios en las industrias internacionales y Harzing (2000) lo aplicó para probar empíricamente y ampliar la tipología de Bartlett y Ghoshal de las multinacionales. Más allá de los enfoques tradicionales de agrupación, se han propuesto otras técnicas diferentes para la segmentación, como "fuzzy cluster", "quality threshold clustering" y "latent cluster". A pesar de sus puntos fuertes, el análisis de conglomerados ha sido criticado por depender de las decisiones de los investigadores, y porque los resultados de la aglomeración pueden no reflejar diferencias reales sino que pueden ser simplemente herramientas estadísticas (Thomas y Venkatraman, 1998). Se aplicó un procedimiento en dos etapas, un agrupamiento jerárquico y un agrupamiento no jerárquico (k-means cluster), método más utilizado. En el procedimiento de agrupamiento jerárquico, utilizamos el método de conglomeración de Ward y como medida de intervalo la distancia euclídea al cuadrado, lo que permite hipotetizar un número apropiado de grupos cuando no hay información disponible a priori. Cuando se han determinado los números de conglomerados como posibles opciones, entonces el método K-medias busca la mejor configuración de los grupos predefinidos colocando observaciones similares entre sí, formando un conglomerado.

Se aplicó un procedimiento en cuatro etapas. Primero se aplicó la técnica de reducción de dimensiones escalamiento óptimo para reducir las variables y medirlas métricamente. Después se realizó un análisis de componentes principales obteniendo 
Estrategia internacional, innovación y rendimiento: conglomerados estratégicos en pymes catalanas / Pinilla y Rialp

seis factores que explican conjuntamente un $64 \%$ de la varianza total. Un tercer paso fue clasificar los factores por medio de un agrupamiento jerárquico, utilizando el método de conglomeración de Ward y como medida de intervalo la distancia euclídea al cuadrado, obteniendo cuatro conglomerados. La consistencia de los factores se demostró mediante la prueba "F" de ANOVA. Por último, con los seis factores y los cuatro conglomerados se realizó un análisis de comparación de medias para darle sentido a cada uno de los conglomerados.

Finalmente, se procedió a cruzar los cuatro conglomerados con las variables de innovación, internacionalización y rendimiento (impacto sobre el desempeño).

Gráfico 1. Resumen metodología utilizada.

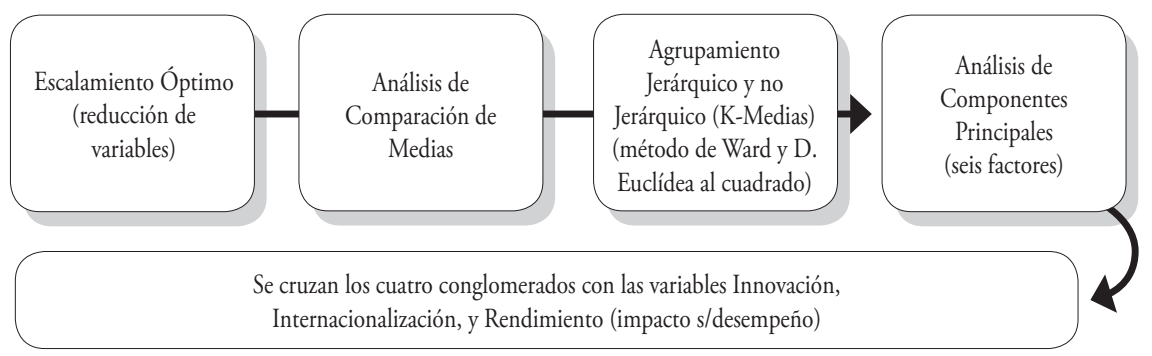

Fuente: elaboración propia.

\section{Medidas de internacionalización}

Para los fines de esta investigación, la internacionalización ha sido considerada como la intensidad de exportación - proporción de las ventas de exportación sobre las ventas totales- (Basile, 2001; Pla-Barber y Alegre, 2007), ya que refleja el grado de internacionalización de una empresa (Sullivan, 1994). La internacionalización se ha medido con el promedio de las exportaciones sobre facturación.

\section{Medidas de innovación}

La innovación se mide por la intensidad en $\mathrm{I}+\mathrm{D}$ (proporción del gasto en $\mathrm{I}+\mathrm{D}$ sobre las ventas totales). Algunos estudios consideran esta medida para medir los esfuerzos de las empresas hacia la innovación (Hirsch y Bijaoui, 1985; McGuinness, 1981), otros han encontrado que tiene un efecto significativo sobre el crecimiento de las pymes (Beise-Zee y Rammer, 2006; Raymond y St-Pierre, 2010). Los mejores resultados son consecuencia: a) del proceso de innovación en la empresa (Freel, 2005; Dibrell et al., 2008), b) de la innovación en el producto por el cual se decide invertir (Freel, 2005; Dibrell et 
al., 2008), y c) de cómo se gestiona ese nuevo producto en términos de marketing y no necesariamente de la cifra del presupuesto en innovación (Lynn et al., 1996). Ar y Baki (2011) hallaron que tanto la innovación en procesos como en productos tienen una asociación fuerte y positiva con el desempeño de una pyme.

\section{Variable de control: tamaño de la empresa / sector económico}

Como variable de control incorporamos el tamaño de la empresa (número total de empleados) que se asocia con los resultados de exportación (Caldera, 2010; Dhanaraj y Beamish, 2003). Uhlaner et al. (2013) demuestran que las empresas de menor tamańo y que innovan en productos suelen estar relacionadas con el crecimiento en las ventas. También se ha incluido la variable de control "sector económico", ya que está directamente relacionada con el éxito del proyecto empresarial.

\section{Resultados empíricos}

Tras realizar un análisis de escalamiento óptimo, se obtuvieron veinte variables, las cuales se utilizaron para realizar un análisis factorial (seis factores). Las variables se etiquetaron para darle sentido e interpretación como muestra la última columna de la tabla 4.

Con los seis factores encontrados y los cuatro conglomerados, se realizó un análisis de comparación de medias (ver tabla 5) para clasificar las pymes catalanas.

\subsection{Conglomerados estratégicos (de análisis cluster)}

Se han identificado cuatro conglomerados estratégicos consistentes de pymes catalanas, cuyo análisis se realiza teniendo presente las tipologías estratégicas de Miles y Snow (1978).

\subsubsection{Cluster 1: "Futuro incierto" (reactores)}

Este conglomerado carece de una clara orientación y estrategia empresarial (reactores). Son empresas con un futuro incierto, carecen de planificación estratégica del producto y de la marca. No hay gestión de proyectos de innovación ni de gestión del conocimiento. Podría tratarse de exportadores reactivos, sin ninguna orientación ni estrategia ni asignación de recursos. Muestran una proporción relativamente baja de las exportaciones con un número limitado de mercados de exportación. Este grupo es el que menor rendimiento tiene en términos de facturación anual y no destaca por sobre los otros grupos en nada. 
Estrategia internacional, innovación y rendimiento: conglomerados estratégicos en pymes catalanas / Pinilla y Rialp

Tabla 4. Matriz de componentes rotada.

\begin{tabular}{|c|c|c|c|c|c|c|c|}
\hline \multirow[b]{2}{*}{ Variables } & \multicolumn{6}{|c|}{ Componente } & \multirow{2}{*}{ OrEst } \\
\hline & 1 & 2 & 3 & 4 & 5 & 6 & \\
\hline Sin plan. estratégica de proyectos & 0,853 & $-0,108$ & 0,177 & 0,072 & $-0,039$ & $-0,029$ & \multirow{6}{*}{ SOE } \\
\hline Sin estrategia de int. tec. ni $\mathrm{I}+\mathrm{D}$ & 0,850 & 0,037 & $-0,085$ & $-0,04$ & 0,097 & $-0,034$ & \\
\hline Sin gestión de proy. de innovación & 0,783 & 0,092 & 0,32 & 0,015 & $-0,155$ & $-0,04$ & \\
\hline Sin ideas ni proyectos & 0,760 & 0,072 & 0,321 & 0,024 & 0,149 & $-0,121$ & \\
\hline Sin gestión del conocimiento & 0,708 & $-0,11$ & $-0,261$ & 0,151 & 0,14 & $-0,141$ & \\
\hline Cultura cerrada a ideas, proy, etc. & 0,704 & $-0,283$ & 0,186 & $-0,015$ & $-0,009$ & 0,164 & \\
\hline Plan. estratégica de proyectos & 0,142 & 0,725 & $-0,02$ & 0,021 & 0,053 & 0,044 & \multirow{6}{*}{$\begin{array}{c}\text { OI, } \\
\text { OM, } \\
\text { OA }\end{array}$} \\
\hline Gestión de proy. de innovación & $-0,095$ & 0,723 & $-0,1$ & $-0,207$ & $-0,056$ & $-0,077$ & \\
\hline Gestión del conocimiento & 0,144 & 0,670 & $-0,129$ & $-0,104$ & 0,283 & 0,245 & \\
\hline Se promueven ideas y creatividad & $-0,021$ & 0,629 & 0,068 & $-0,032$ & 0,183 & 0,042 & \\
\hline Cultura abierta a ideas y proyectos & $-0,365$ & $-0,532$ & 0,162 & 0,32 & $-0,041$ & 0,177 & \\
\hline Marketing (posic. marca) & 0,134 & $-0,526$ & $-0,288$ & 0,38 & $-0,063$ & 0,071 & \\
\hline Procesos internos flexibles & $-0,212$ & 0,008 & $-0,752$ & 0,064 & 0,192 & 0,124 & \multirow{3}{*}{ SOE } \\
\hline Sin planificación del producto & 0,307 & 0,176 & 0,675 & $-0,18$ & 0,109 & 0,074 & \\
\hline Sin marketing (posic. marca) & 0,37 & $-0,086$ & 0,600 & 0,039 & 0,02 & 0,129 & \\
\hline Bajo éxito en proy. sin subv. púb. & $-0,215$ & $-0,061$ & $-0,165$ & 0,731 & 0,094 & 0,076 & \multirow{2}{*}{$\mathrm{OP}$} \\
\hline Plan. estratégica del prod. y de TIC & 0,373 & $-0,171$ & 0,049 & 0,584 & $-0,282$ & $-0,036$ & \\
\hline Alto éxito en proy., subv. pública & 0,035 & $-0,038$ & $-0,142$ & $-0,106$ & 0,797 & $-0,233$ & \multirow{2}{*}{$\mathrm{OE}$} \\
\hline Gestión de I+D y de TTI & $-0,125$ & 0,429 & 0,266 & 0,193 & 0,532 & 0,21 & \\
\hline Procesos internos inflexibles & 0,025 & $-0,121$ & 0,013 & 0,069 & $-0,131$ & 0,908 & SOE \\
\hline
\end{tabular}

OrEst: orientación estratégica, $\mathrm{SOE}$ : sin OrEst, OI: orientación alainnovación, OM: orientación al mercado. OA: orientación al aprendizaje, OP: orientación al producto, OE: orientación al emprendimiento. Fuente: elaboración propia.

\subsubsection{Cluster 2: "Cómoda posición en el mercado" (defensores)}

Las empresas de este conglomerado se pueden definir como defensoras por su clara orientación al producto (planificación del ciclo de vida y desarrollo) y al mercado (portafolio diferenciado y alta calidad de productos, creación y mantenimiento de valor para el cliente). Es el conglomerado que concentra más empresas. Muestran en general una planificación estratégica de los productos y de las TIC y descartan subvenciones públicas para impulsar proyectos de innovación. Es el segundo conglomerado que más gasto tiene sobre la facturación en I+D. Su proporción media de las exportaciones hace pensar que podrían tener una estrategia y asignación de recursos en búsqueda de mercados de 
exportación para internacionalizarse. En este grupo se aprecia una actitud estratégica que va de la mano con un alto rendimiento medido en términos de facturación anual, la más alta de los cuatro conglomerados.

Tabla 5. Comparación de medias entre clusters y factores.

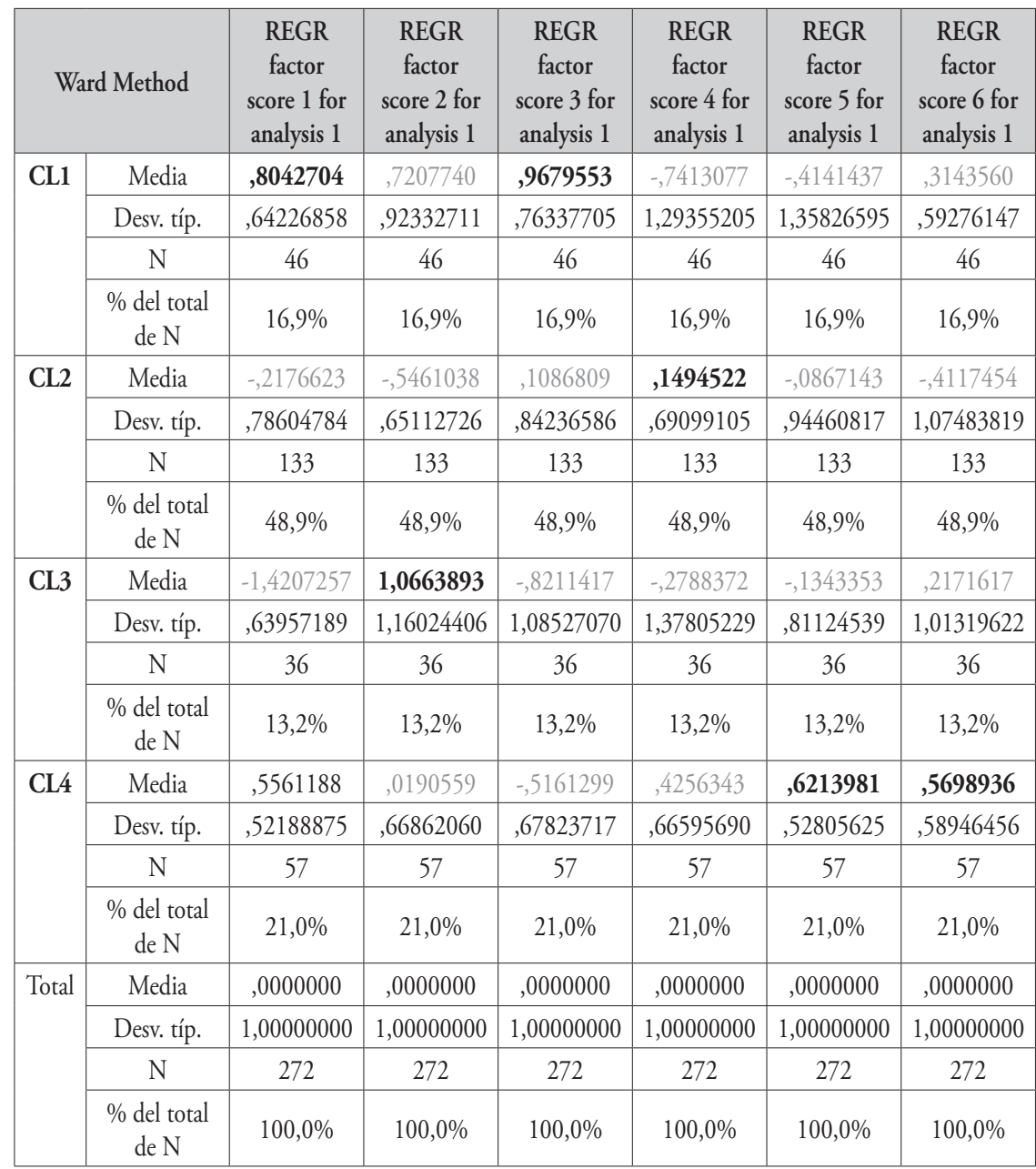

Fuente: elaboración propia.

\subsubsection{Cluster 3: "Apuesta por lo nuevo y por el cambio" (exploradores)}

El tercer clúster se ha llamado exploradores, ya que es un conglomerado que pone énfasis en la OI y el emprendimiento. Son empresas con una gran capacidad de innovación, 
Estrategia internacional, innovación y rendimiento: conglomerados estratégicos en pymes catalanas / Pinilla y Rialp

asumen riesgos y son proactivas. Además, realizan gestión de proyectos de innovación, gestión del conocimiento y promueven ideas y la creatividad para los proyectos de innovación. La OI es liderada por la alta dirección y disponen de un sistema de control de calidad para sus productos y servicios. Existe un procedimiento formal para seleccionar las mejores ideas (futuros proyectos de innovación), dedicándoles tiempo y recursos. Realizan estudios de mercado y utilizan técnicas avanzadas de creatividad. Es el conglomerado que más gasta en $\mathrm{I}+\mathrm{D}$ de los cuatro y tienen la segunda relación de exportación más alta de todos, lo que lo hace depender en gran medida de la internacionalización. La OI y la capacidad de gestión, junto con la propensión a la internacionalización lleva a que estas empresas tengan el segundo mejor rendimiento económico de los cuatro conglomerados, medido por el promedio de la facturación anual.

La tabla 6 muestra los perfiles de cada conglomerado.

Tabla 6. Perfiles de los clister.

\begin{tabular}{|c|c|c|c|c|}
\hline & CL1 & CL2 & CL3 & CL4 \\
\hline No de casos & $\mathrm{N}=46$ & $\mathrm{~N}=133$ & $\mathrm{~N}=36$ & $N=57$ \\
\hline$\%$ s $/$ la muestra & $17 \%$ & $49 \%$ & $13 \%$ & $21 \%$ \\
\hline Tamańo de la PYME & pequeña & mediana & pequeña & pequeña \\
\hline $\begin{array}{l}\text { No de trabajadores } \\
\text { (promedio) }\end{array}$ & 25 & 54 & 42 & 32 \\
\hline Industrias más relevantes & $\begin{array}{l}\text { Metalúrgica, } \\
\text { productos equipos } \\
\text { metálicos, química, } \\
\text { otros }\end{array}$ & $\begin{array}{c}\text { TIC y } \\
\text { telecomunicaciones, } \\
\text { metalúrgica, } \\
\text { equipos eléctricos } \\
\text { y electrónicos, } \\
\text { construcción, } \\
\text { química }\end{array}$ & $\begin{array}{l}\text { Química, servicios } \\
\text { sanitarios }\end{array}$ & $\begin{array}{l}\text { Metalúrgica, TIC y } \\
\text { telecomunicaciones }\end{array}$ \\
\hline $\begin{array}{l}\text { Innovación (promedio } \\
\% \text { gastos en I+D sobre } \\
\text { facturación) }\end{array}$ & $7 \%$ & $9 \%$ & $19 \%$ & $6 \%$ \\
\hline $\begin{array}{l}\text { Internacionalización } \\
\text { (promedio de las } \\
\text { exportaciones sobre } \\
\text { facturación) }\end{array}$ & $15 \%$ & $23 \%$ & $27 \%$ & $31 \%$ \\
\hline $\begin{array}{l}\text { Rendimiento (promedio } \\
\text { facturación anual en } \\
\text { millones de euros) }\end{array}$ & 3,81 & 11,97 & 8,11 & 5,43 \\
\hline
\end{tabular}

Fuente: elaboración propia.

\subsubsection{Cluster 4: "Mentalidad abierta a nuevos desafíos" (analizadores)}

El cuarto cluster es un conglomerado proactivo estratégicamente y se ha llamado analizadores por estar relacionado con la estrategia de internacionalización, ya que, dentro 
de sus prácticas de gestión empresarial hay una estrategia de internacionalización tecnológica (transferencia tecnológica internacional - TTI) y de I+D. La cartera de proyectos supone la incorporación de conocimiento tecnológico en el mercado (nuevas tecnologías e I+D). Se utilizan subvenciones públicas para proyectos de innovación. Estas empresas son las más exportadoras de los cuatro conglomerados y así lo demuestra el promedio de las exportaciones sobre facturación.

Los temas de crecimiento internacional pueden estar relacionados con la búsqueda de oportunidades y la toma de riesgos, dada la mayor incertidumbre y los riesgos de expansión internacional (Yli-Renko et al., 2002). Así, este grupo tiene un enfoque hacia los mercados extranjeros.

\section{Discusión}

Los resultados proporcionan evidencia de perfiles y patrones estratégicos de pymes distintos y comparten muchos puntos en común con las tipologías de gestión y marketing estratégico encontrados para grandes empresas. Así, por ejemplo, se tiene el conglomerado OP y OM que comparte algunas características definidas por Miles y Snow (1978) donde esas empresas, al igual que las nuestras, han sido descritas como defensoras, poniendo énfasis en el desarrollo de nuevos productos y servicios. Miles y Snow definieron una tipología de "reactores" como nuestro conglomerado "futuro incierto", ya que ambos carecen de un perfil estratégico consistente. Nuestro trabajo ha encontrado conglomerados relacionados con el comportamiento de las pymes catalanas orientadas a la internacionalización y al marketing e innovación. En sus estudios, Miles y Snow (1978 y 1986) muestran una actitud de las empresas más o menos proactiva hacia el entorno externo. En este caso, se encontró un conglomerado cuya orientación es al producto y otro con una orientación internacional. Estas empresas orientadas hacia el exterior tienen una actitud innovadora-proactiva.

El conglomerado que muestra una estrategia de internacionalización es el único que hace hincapié en la incorporación de conocimiento tecnológico con un enfoque en los mercados extranjeros. Tanto el conglomerado con orientación internacional como el orientado a la innovación y emprendimiento tienen un alto grado de orientación internacional. Estas actitudes y características, representadas por un porcentaje de exportación sobre ventas, así como el compromiso hacia la internacionalización, han hecho hincapié en las influencias de los resultados de exportación (Aaby y Slater, 1989). La probabilidad de tener un alto desempeño en los mercados extranjeros para los conglomerados orientados al producto/mercado, a la innovación/emprendimiento y con orientación internacional es mayor que el de las empresas que carecen de orientación estratégica. Las empresas de los tres últimos conglomerados se caracterizan por un ma- 
Estrategia internacional, innovación y rendimiento: conglomerados estratégicos en pymes catalanas / Pinilla y Rialp

yor porcentaje de exportación sobre facturación, lo que ratifica su participación en el mercado internacional y de su desempeño internacional. Por tanto, nuestros resultados se condicen con los hallazgos de Miles y Snow y con aquellas investigaciones sobre orientación estratégica en mercados domésticos, que sostienen que las empresas con perfiles estratégicos claros obtienen un mejor desempeño que las que no lo presentan (Cano et al., 2004; Miles y Snow, 1978; Shoham et al., 2005).

\section{Conclusiones}

Este trabajo pretende contribuir a la discusión sobre las estrategias de crecimiento de las pymes y su interdependencia con perfiles empresariales. Este estudio confirma una relación positiva entre orientaciones estratégicas y rendimiento, expresado como el promedio de facturación anual. Tres de los cuatro conglomerados identificados en la muestra, a saber, producto/mercado, innovación/emprendimiento y estrategia de internacionalización persiguen más activamente oportunidades internacionales, crecer más rápidamente, y exhiben un rendimiento superior en comparación con el primer conglomerado representado por aquellas empresas que carecen de una estrategia clara. Lo anterior no hace más que confirmar los hallazgos de estudios sobre iniciativa empresarial internacional que ponen de manifiesto la relación orientación / crecimiento como la base de un rendimiento superior, desafiando las formas tradicionales de expansión internacional (Oviatt y McDougall, 1994). Los resultados son relevantes para los directivos y empresarios para situar su negocio estratégicamente, para los responsables políticos haciéndoles ver que las pymes que carecen de una visión y orientación estratégica deberían tener primero una asesoría estratégica en lugar de medidas específicas de apoyo a la exportación.

Pero esta investigación también tiene algunas limitaciones. La base de datos se limita sólo a pymes catalanas, cuenta con 17 sectores económicos y el análisis puede ser sujeto a sesgos específicos. Los conglomerados representan una simplificación de la realidad empresarial, una simplificación que es útil para la clasificación de empresas y entender su comportamiento internacional. La innovación y la internacionalización son procesos que evolucionan con el tiempo, por lo que nuestro análisis no puede capturar la evolución de las empresas de un tipo de conglomerado a otro en el tiempo (son datos de corte transversal). Las investigaciones futuras podrían tratar de confirmar y afinar estos conglomerados estratégicos con el fin de verificar si se mantienen en un área geográfica más extensa. Sin duda que una investigación longitudinal de análisis clúster de pymes podría aportar datos interesantes sobre el desarrollo y el comportamiento de la internacionalización a largo plazo y las consecuencias relacionadas con el rendimiento. 


\section{Bibliografía}

Aaby, N.E. y Slater, S. F. (1989). Management influences of export performance: a review of the empirical literature 1978-1988. International Marketing Review, 6(4), 7-26.

Almeida, R. y Fernandes, A. M. (2008). Openness and technological innovations in developing countries: evidence from Firm-Level surveys. Journal of Development Studies, 44(5), 701-727.

Andersson, M. y Loof, H. (2011). Small business innovation: firm level evidence from Sweden. The Journal of Technology Transfer, 37(5), 732-754.

Ansoff, I. (1957). Strategies for diversification. Harvard Business Review, 35(5), 113-124. Amar, A. y Juneja, J. (2008). A descriptive model of innovation and creativity in organizations: a synthesis of research and practice. Knowledge Management Research \& Practice, 6(4), 298-311.

Añon, D. y Driffield, A. N. (2011). Exporting and innovation performance: analysis of the annual small business survey in the UK. International Small Business Journal, 29(1), 4-24.

Aragón, A.; Sánchez, G. y Mueses, A. (2015). Gestión del conocimiento y resultados empresariales: efecto mediador de las prácticas de recursos humanos. Revista Europea de Dirección y Economía de la Empresa, http://dx.doi.org/10.1016/j. redee.2015.03.003.

Ar, I. M. y Baki, B. (2011). Antecedents and performance impacts of product versus process innovation: empirical evidence from SMEs located in Turkish science and technology parks. European Journal of Innovation Management, 14(2), 172-206.

Armario, J. M.; Ruiz, D. M. \& Armario, E. M. (2008). Market orientation and internationalization in small and medium-sized enterprises. Journal of Small Business Management, 46(4), 485-511.

Bahadir, S. C.; Bharadwaj, S. y Parzen, M. (2009). A meta-analysis of the determinants of organic sales growth. International Journal of Research in Marketing, 26(4), 263-275.

Baker, W. y Sinkula, J. (1999a). The synergistic effect of market orientation on organizational performance. Journal of the Academy of Marketing Science, 27(4), 411-27.

Baker, W. y Sinkula, J. (1999b). Learning orientation, market orientation, and innovation: integrating and extending models of organizational performance. Journal of Market Focused Management, 4(4), 295-308. 
Estrategia internacional, innovación y rendimiento: conglomerados estratégicos en pymes catalanas / Pinilla y Rialp

Balabanis, G. I. \& Katsikeas, E. (2003). Being an entrepreneurial exporter: does it pay? International Business Review, 12(2), 233-252.

Bannò, M.; Piscitello, L. y Amorim Varum, C. (2013). The impact of public support on SMEs' Outward FDI: evidence from Italy. Journal of Small Business Management, 52(1), 22-38.

Barney, J. (1991). Firm resources and sustained competitive advantage. Journal of Management, 17(1), 99-120.

Basile, R. (2001). Export behaviour of Italian manufacturing firms over the nineties: the role of innovation. Research Policy, 30(8), 1185-1201.

Becker, W. y Dietz, J. (2004). R\&D cooperation and innovation activities of firms evidence for the German manufacturing industry. Research Policy, 33(2), $209-223$.

Becker, S. O. y Egger, P. H. (2007). Endogenous product versus process innovation and a firm's propensity to export. Empirical Economics, 44(1), 1-26.

Beise-Zee, R. y Rammer, C. (2006), Local user-producer interaction in innovation and export performance of firms. Small Business Economics, 27, 207-222.

Bell, J.; Crick, D. y Young, S. (2004). Small firm internationalisation and business strategy: an exploratory study of "knowledge intensive" and "traditional" manufacturing firms in the UK. International Small Business Journal, 22(1), $23-56$.

Berthon, P.; Hulbert, J. M. y Pitt, L. (2004). Innovation or customer orientation? An empirical investigation. European Journal of Marketing, 38(9/10), 1065 1090.

Bilkey, W. J. y Tesar, G. (1977). The export behaviour of smaller Wisconsin manufacturing firms. Journal of International Business Studies, 9, 93-98.

Brewer, P. (2009). Australia’s export promotion program: is it effective? Australian Journal of Management, 34(1), 125-142.

Buzzel, R. D. y Gale, B. T. (1987). The PIMS principle: linking strategy to performance. New York: The Free Press.

Cadogan, J. W.; Cui, C. C. y Li, E. K. Y. (2003). Export market oriented behaviour and export performance. International Marketing Review, 20, 493-513.

Calantone, R. J.; Cavusgil, S. T. y Zhao, Y. (2002). Learning orientation, firminnovation capability, and firm performance. Industrial Marketing Management, 31(6), 515-524.

Caldera, A. (2010). Innovation and exporting: evidence from Spanish manufacturing firms. Review of World Economics, 146(4), 657-689. 
Cano, C. R.; Carrillat, F. A. y Jaramillo, F. (2004). A meta-analysis of the relationship between market orientation and business performance: evidence from five continents. International Journal of Research in Marketing, 21(2), 179 - 200.

Cassiman, B. y Golovko, E. (2011). Innovation and internationalization through exports. Journal of International Business Studies, 42(1), 56-75.

Cassiman, B. y Martinez-Ros, E. (2007). Product innovation and exports: Evidence from Spanish manufacturing. IESE Working Paper, mimeo.

Caruana, A.; Morris, M. H. y Vella, A. J. (1998). The effect of centralization and formalization on entrepreneurship in export firms. Journal of Small Business Management, 31(1), 16-29.

Conant, J. S.; Mokwa, M. P. y Varadarajan, P. R. (1990). Strategic types, distinctive marketing competencies and organizational performance: a multiple measuresbased study. Strategic Management Journal, 11, 365-383.

Cuervo-Cazurra, A.; Maloney, M. y Manrakhan, S. (2007). Causes of difficulty in internationalization. Journal of International Business Studies, 38(5), 709-725.

Day, G. S. y Wensley, R. (1988). Assessing advantage: a framework for diagnosing competitive superiority. Journal of Marketing, 52(4), 1-20.

D’Angelo, A. (2012). Innovation and export performance: a study of Italian high-tech SMEs. Journal of Management \& Governance, 16(3), 393-423.

Dess, G. G. y Davis, P. S. (1984). Porter's (1980) generic strategies as determinants of strategic group membership and organizational performance. Academy of Management Journal, 27(3), 467-488.

Dhanaraj, C. y Beamish, P. W. (2003). A resource-based approach to the study of export performance. Journal of Small Business Management, 41(3), 242-261.

Damijan, J. P.; Kostevc, Ä. y Polanec, S. (2010). From innovation to exporting or vice versa? The World Economy, 33(3), 374-398.

Darroch, J. y McNaughton, R. (2002), Examining the link between knowledge management practice and types of innovation. Journal of Intellectual Capital, 3(3), 210-222.

Denicolai, S.; Hagen, B. y Pisoni, A (2015). Be international or be innovative? Be Both? The Role of the Entrepreneurial Profile. Journal of International Entrepreneurship, published online: 17 March 2015 (forthcoming).

Delmar, F.; Davidsson P. y Gartner W. (2003), Arriving at the high-growth firm. Journal of Business Venturing, 18, 189-216.

Dibrell, C.; David, P. y Craig, J. (2008). Fueling innovation through information technology in SMEs. Journal of Small Business Management, 46(2), 203-218. 
Estrategia internacional, innovación y rendimiento: conglomerados estratégicos en pymes catalanas / Pinilla y Rialp

Drucker, P. F. (1954). The practice of management. New York: Harper and Row.

Eddleston, K.; Kellermans, F. y Sarathy, R. (2008). Resource configuration in family firms: linking resources, strategic planning and technological opportunities to performance. Journal of Management Studies, 45(1), 26-50.

Ernst, D. y Unctad (2005). Complexity and internationalisation of innovation: why is chip design moving to Asia? International Journal of Innovation Management, 9(1), 47-73.

Fernández-Mesa, A. y Alegre, J. (2015). Entrepreneurial orientation and export intensity: examining the interplay of organizational learning and innovation. International Business Review, 24(1), 148-156.

Fritz, W. (1996). Market orientation and corporate success: findings from Germany. European Journal of Marketing, 30(8), 59-74.

Furman, J.; Porter, M. y Stern, S. (2002). The determinants of national innovative capacity. Research Policy, 31(6), 899-933.

Frambach, R. T.; Prabhu, J. y Verhallen, T. M. M. (2003). The influence of business strategy on new product activity: the role of market orientation. International Journal of Research in Marketing, 20(4), 377-397.

Freel, M. S. (2005). Patterns of innovation and skills in small firms. Technovation, 25(2), 123-134.

Gatignon, H. y Xuereb, J. M. (1997). Strategic orientation of the firm and new product performance. Journal of Marketing Research, 34, 77-90.

Golovko, E. y Valentini, G. (2011). Exploring the complementarity between innovation and export for SMEs' growth. Journal of International Business Studies, 42(3), 362-380.

Hagen, B.; Zucchella, A.; Cerchiello, P. y De Giovanni, N. (2012). International strategy and performance-clustering strategic types of SMEs. International Business Review, 21, 369 -382.

Halilem, N.; Nabil, A. y Réjean, L. (2014). Exploring the relationships between innovation and internationalization of small and mediumsized enterprises: a nonrecursive structural equation model. Canadian Journal of Administrative Sciences, 31(1), 18-34.

Han, J. K.; Kim, N. y Srivastava, R. K. (1998). Market orientation and organizational performance: Is innovation a missing link? Journal of Marketing, 62(4), $30-45$.

Harzing, A. W. (2000a). Cross-national mail surveys: why do response rates differ between countries? Industrial Marketing Management, 29(3), 243-254. 
Harzing, A. W. (2000b). An empirical analysis and extension of the Bartlett and Ghoshal typology of multinational companies. Journal of International Business Studies, 31(1), 101-120.

Henderson, J. y Weiler, S. (2010). Entrepreneurs and job growth: probing the boundaries of time and space. Economic Development Quarterly, 24(1), 23-32.

Helfat, C. E. (2007). Dynamic capabilities: understanding strategic change in organizations, Blackwell.

Hessels, S. (2007). Innovation and international involvement of Dutch SMEs. International Journal of Entrepreneurship and Small Business, 4(3), 234-255.

Hirsch, S. y Bijaoui, I. (1985). R\&D intensity and export performance - a micro view, Weltwirtschaftliches Archiv. Review of World Economics, 121(2), 238-251.

Homburg, C.; Krohmer, H. y Workman, J. P. Jr. (2004). A strategy implementation perspective of market orientation. Journal of Business Research, 1331-1340.

Huber, G. P. (1991). Organizational learning: the contributing processes and the literatures. Organization Science, 2(1), 88-115.

Hult, G. T. M.; Hurley, R. F. y Knight, G. A. (2004). Innovativeness: its antecedents and impact on business performance. Industrial Marketing Management, 33, 429-438.

Human, G. y Naudé, P. (2009). Relationship or Innovation orientation in B2B markets: empirical results from an emerging market. En the 25th IMP-Conference. p. 1-17.

Hunt, S. D. y Morgan, R. M. (1995). The comparative advantage theory of competition. Journal of Marketing, 59, 1-15.

Hurley, R. F. y Hult, G. T. M. (1998). Innovation, market orientation and organizational learning: an integration and empirical examination. Journal of Marketing, 62(Julio), $42-54$.

Jaworski, B. J. y Kohli, A. K. (1993). Market orientation: antecedents and consequences. Journal of Marketing, 57(3), 53-70.

Kafouros, M. I.; Buckley, P. J.; Sharp, J. A. y Wang, C. (2008). The role of internationalization in explaining innovation performance. Technovation, 28(1-2), 63-74.

Keh, H. T.; Nguyen, T. T. M. y Ng, H. P. (2007). The effects of entrepreneurial orientation and marketing information on the performance of SMEs. Journal of Business Venturing, 22(4), 592-611.

Ketchen, D. J. y Shook, C. L. (1996). The application of cluster analysis in strategic management research: an analysis and critique. Strategic Management Journal, 17, 441- 458. 
Estrategia internacional, innovación y rendimiento: conglomerados estratégicos en pymes catalanas / Pinilla y Rialp

Khilji, S.; Mroczkowski, T. y Bernstein, B. (2006). From invention to innovation: toward developing an integrated innovation model for biotech firms. Journal of Product Innovation Management, 23, 528-540.

Knight, G. y Liesch, P. (2002). Information internalisation in internationalising the firm. Journal of Business Research 55(12), 981-995.

Kirca, A. H.; Cavusgil, S. T. y Hult, G. T. M. (2009). The effects of national culture on market orientation: conceptual frameworks and research propositions. International Business Review, 18(2), 111-118.

Kirca, A. H.; Jaycachandran, S. y Bearden, W. O. (2005). Market orientation: a metaanalytic review and assessment of its antecedents and impact on performance. Journal of Marketing, 69(2), 24-41.

Kohli, A. K. y Jaworski, B. J. (1990). Market orientation: the construct, research propositions, and managerial implications. Journal of Marketing, 54(2), 1-18.

Kohli, A. K.; Jaworski, B. J. y Kumar, A. (1993). MARKOR: a measure of market orientation. Journal of Marketing Research, 30(4), 467-477.

Kotabe, M.; Dunlap-Hinkler, D.; Parente, R. y Mishra, H. A. (2007). Determinants of cross-national knowledge transfer and its effect on firm innovation. Journal of International Business Studies, 38(2), 259-282.

Kotler, P. (2002). Marketing management: analysis, planning and control. New York: Prentice Hall.

Kuivalainen, O.; Sundqvist, S. y Servais, P. (2007). Firms' degree of born-globalness, international entrepreneurial orientation and export performance. Journal of World Business, 42, 253-267.

Larimo, J. (2007). Different types of exporting SMEs: Similarities and differences in export performance. International Marketing Research, 17, 17-62.

Leonidou, L. y Katsikeas, C. (1996). The export development process: an integrative review of empirical models. Journal of International Business Studies, 27, 517-552.

Li, Y.; Zhao, Y.; Tan, J. y Liu, Y. (2008). Moderating effects of entrepreneurial orientation on market orientation-performance linkage: evidence from Chinese small firms. Journal of Small Business Management, 46(1), 113-133.

Liebowitz, J. (2008). Two forgotten elements of a knowledge management strategy. Knowledge Management Research \& Practice, 6(3), 239-244.

Liu, S.; Luo, X. y Shi, Y. Z. (2002). Integrating customer orientation, corporate entrepreneurship, and learning orientation in organizations-in-transition: an empirical study. International Journal of Research in Marketing, 19, 367-82. 
Lumpkin, G. T. y Dess, G. G. (1996). Clarifying the entrepreneurial orientation construct and linking it to performance. Academy of Management Review, 21(1), 135-172.

Lynn, G. S.; Morone, J. G. y Paulson, A. S. (1996). Marketing and discontinuous innovation: the probe and learn process. California Management Review, 38(3), 8-37.

Lundvall, B. A. (2007). National innovation systems - analytical concept and development tool. Industry and Innovation, 14(1), 95-119.

Madrid, A.; Garcia, D. y Van Auken, H. (2009). Barriers to innovation among Spanish manufacturing SMEs. Journal of Small Business Management, 47(4), 465-488.

Madsen, T. K. (1989). Successful export marketing management: some empirical evidence. International Marketing Review, 6(4), 41-57.

March-Chorda, I.; Gunasekaran, A. y Lloria-Aramburo, B. (2002). Product development process in Spanish SME's: an empirical research. Technovation, 22(5), 301-312.

Marlin, D.; Ketchen, D. y Lamont, B. (2007). Equifi nality and the strategic groupsperformance relationship. Journal of Managerial Issues, 19(2), 208-233.

McKee, D. O.; Varadarajan, P. R. \& Pride, W. M. (1989). Strategic adaptability and firm performance: a market-contingent perspective. Journal of Marketing, 53(3), 21-35.

McGuinness, N. W. y Little, B. (1981). The impact of R\&D spending on the foreign sales of new Canadian industrial products. Research Policy, 10(1), 78-98.

Melin, L. (1992). Internationalisation as a strategy process. Strategic Management Journal, 13, 99-118.

Miles, R. E. y Snow, C. C. (1978). Organizational strategy, structure, and process. New York: McGraw-Hill Book Company.

Miles, R. E. y Snow, C. C. (1986). Organizations: new concepts for new forms. California Management Review, 28(3), 66-73.

Moen, O. (1999). The relationship between firm size, competitive advantages and export performance revised. International Small Business Journal, 18(1), 53-72.

Monreal-Pérez, J.; Aragón-Sánchez, A. y Sánchez-Marín, G. (2012). A longitudinal study of the relationship between export activity and innovation in the Spanish firm: the moderating role of productivity. International Business Review, 21(5), 862-877.

Morgan, N. A.; Vorhies, D. W. y Mason, C. H. (2009). Market orientation, marketing capabilities, and firm performance. Strategic Management Journal, 30, 909-920. 
Estrategia internacional, innovación y rendimiento: conglomerados estratégicos en pymes catalanas / Pinilla y Rialp

Morrison, A. J. y Roth, K. (1992). A taxonomy of business-level strategies in global industries. Strategic Management Journal, 13, 399-418.

Narver, J. C. y Slater, S. F. (1990). The effect of a market orientation on business profitability. Journal of Marketing, 54(4), 20-35.

Nauwelaerts, Y. y Vijfeyken, E. (2013). 6. The effect of export promotion programs on export satisfaction: a study in the Flemish design sector. In De Beule, F. \& Nauwelaerts, Y. (Eds.) Innovation and creativity: Pillars of the future global economy, Edward Elgar Publishing Ltd: Cheltenham, UK, 126-154.

Nieto, M. J. y Santamaría, L. (2010). Technological collaboration: bridging the innovation gap between small and large firms. Journal of Small Business Management, 48(1), 44-69.

Noble, C. H.; Sinha, R. K. y Kumar, A. (2002). Market orientation and alternative strategic orientations: a longitudinal assessment of performance implications. Journal of Marketing, 66, 25-39.

Oke, A.; Burke, G. y Myers, A. (2007). Innovation types and performance in growing UK SMEs. International Journal of Operations \& Production Management, 27 (7), 735-753.

Oviatt, B. M. y McDougall, P. P. (1994). Toward a theory of international new ventures. Journal of International Business Studies, 25(1), 45-64.

Palacios, D.; Gil, I. y Garrigos, F. (2009). The impact of knowledge management practices on innovation and entrepreneurship in the biotechnology and telecommunications industries. Small Business Economics, 32, 291-301.

Pelham, A. M. y Wilson, D. T. (1996). A longitudinal study of the impact of market structure, firm structure, strategy, and market orientation culture on dimensions of small-firm performance. Journal of the Academy of Marketing Science, 24, 27-43.

Pla-Barber, J. y Alegre, J. (2007). Analysing the link between export intensity, innovation and firm size in a science-based industry. International Business Review, 16(3), 275-293.

Polanyi, M. (1966). The tacit dimension. Routledge and Kegan Paul: London, UK.

Porter, M. E. (1980). Competitive strategy. New York: The Free Press.

Porter, M. (1987). From competitive advantage to corporate strategy. Harvard Business Review. May-June (3), 43-59.

Prashantham, S. (2008). New venture internationalization as strategic renewal. European Management Journal, 26(6), 378-387. 
Pleshko, L. y Soulden, N. (2003). The profit effects of product-market growth strategy: a financial services example. Journal of Financial Services Marketing, 7(3), 258-266.

Raymond, L.; St-Pierre, J. (2010). R\&D as a determinant of innovation in manufacturing SMEs: an attempt at empirical clarification, Technovation, 30(1), 48-56.

Robertson, C. y Chetty, S. K. (2000). A contingency-based approach to understanding export performance. International Business Review, 9(2), 211-235.

Roper, S.; Du, J. y Love, J. H. (2008). Modelling the innovation value chain. Research Policy, 37(6), 961-977.

Ruzzier, M.; Hisrich, R. D. y Antoncic, B. (2006). SME internationalization research: past, present and future. Journal of Small Business and Enterprise Development, 13(4), 476-497.

Sapienza, H. J.; Autio, E.; George, G. y Zahra, S. A. (2006). A capabilities perspective on the effects of early internationalization on firm survival and growth. Academy of Management Review, 31(4), 914-933.

Schubert, T. y Simar, L. (2010). Innovation and export activities in the German mechanical engineering sector: an application of testing restrictions in production analysis. Journal of Productivity Analysis, 36(1), 55-69.

Schumpeter, J. (1934). The theory of economic development. Harvard University Press, Cambridge, MA.

Schumpeter, J. (1942). Capitalism, socialism and democracy. Harper and Row, New York.

Shoham, A.; Rose, G. y Kropp, F. (2005). Market orientation and performance: a meta-analysis. Marketing Intelligence and Planning, 23(4/5), 435-454.

Simpson, P. M.; Siguaw, J. A. y Enz, C. A. (2006). Innovation orientation outcomes: the good and the bad. Journal of Business Research, 59, 1133-1141.

Sinkula, J. M. (1994). Market information processing and organizational learning. Journal of Marketing, 58(1), 35-45.

Sinkula, J. M.; Baker, W. E. y Noordewier, T. (1997). A framework for market-based organizational learning: linking values, knowledge and behavior. Journal of the Academy of Marketing Science, 25(4), 305-318.

Slater, S. F. y Narver, J. C. (1995). Market orientation and the learning organization. Journal of Marketing, 59(3), 63-67.

Smart, D. T. y Conant, J. S. (1994). Entrepreneurial orientation, distinctive marketing competencies and organizational performance. Journal of Applied Business Research, 10(3), 28-38. 
Estrategia internacional, innovación y rendimiento: conglomerados estratégicos en pymes catalanas / Pinilla y Rialp

Snowden, D. (2003). Innovation as an objective of knowledge management. Part I: the landscape of management. Knowledge Management Research \& Practice, 1(2), 113-119.

Speed, R. (1993). Maximizing the potential of strategic typologies for marketing strategy research. Journal of Strategic Marketing, 1, 171-188.

Stock, R. M. y Zacharias, N. A. (2011). Patterns and performance outcomes of innovation orientation. Academy of Marketing Science, 39, 870-888.

Sullivan, D. (1994). Measuring the degree of internationalization of a firm. Journal of International Business Studies, 25(2), 325-342.

Tether, B. (2002). Who co-operates for innovation, and why - an empirical analysis. Research Policy, 31(6), 947-967.

Thomas, H. y Venkatraman, N. (1998). Research on strategic groups: progress and prognosis. Journal of Management Studies, 25, 537-555.

Uhlaner, L. M.; Van Stel, A.; Duplat, V. y Zhou, H. (2013). Disentangling the effects of organizational capabilities, innovation and firm size on SME sales growth. Small Business Economics, 41(3), 581-607.

Van Beveren, I. y Vandenbussche, H. (2010). Product and process innovation and firms' decision to export. Journal of Economic Policy Reform, 13(1), 3-24.

Verhees, F. y Meulenberg, M. (2004). Market orientation, innovativeness, product innovation and performance in small firms.

Vila, N. y Kuster, I. (2007). The importance of innovation in international textile firms. European Journal of Marketing, 41(1), 17-36.

Wiklund, J. y Shepherd, D. (2005). Entrepreneurial orientation and small business performance: a configurational approach. Journal of Business Venturing, 20(1), 71-91.

Woerter, M. \& Roper, S. (2010). Openness and innovation. Home and export demand effects on manufacturing innovation: panel data evidence for Ireland and Switzerland. Research Policy, 39(1), 155-164.

Wood, V. R. y Robertson, K. R. (1997). Strategic orientation and export success: an empirical study. International Marketing Review, 14(6), 424-444.

Woodside, A. G.; Sullivan, D. P. y Trappey, R. J. (1999). Assessing relationships among strategic types, distinctive marketing competencies and organizational performance. Journal of Business Research, 45(2), 135-146.

Wright, P.; Kroll, M. J.; Pray, B. \& Lado, A. (1995). Strategic orientations, competitive advantage, and business performance. Journal of Business Research, 33(2), $143-151$. 
Yannopoulos, P. (2010). Export assistance programs: Insights from Canadian SMEs. International Review of Business Research Papers, 6(5), 36-51.

Yi, J.; Wang, Ch. y Kafouros, M. (2013). The effects of innovative capabilities on exporting: do institutional forces matter? International Business Review, 22(2), 392-406.

Yli-Renko, H.; Autio, E. y Tontti, V. (2002). Social capital, knowledge, and the international growth of technology-based new firms. International Business Review, 11(3), 279-304.

Yongho, L.; Juneseuk, S. y Yonktae, P. (2012). The changing pattern of SME's innovativeness through business model globalization. Technological Forecasting \& Social Change, 79(5), 832-842.

Yu, X. y Si, S. (2012). Innovation, internationalization and entrepreneurship: a new venture research perspective. Innovation: Management, policy \& practice, 14(4), 524-539.

Zahra, S.; Ireland, R. y Hitt, M. (2000). International expansion by new venture firms: international diversity, mode of market entry, technological learning and performance. Academy of Management Journal, 43(5), 925-950.

Zheng, K.; Yong, G.; Yang, Z. y Zhou, N. (2005). Developing strategic orientation in China: antecedents and consequences of market and innovation orientations. Journal of Business Research, 58, 1049-1058.

Zhou, Z. K.; Yim, K. C. y Tse, D. (2005). The effects of strategic orientations on technology -and market- based breakthrough innovations. Journal of Marketing, 69(1), 42-60.

Zhou, Z. K. y Li, C. B. (2007). How does strategic orientation matter in Chinese firms? Asia Pacific Journal of Management, 24, 447-466.

\section{Cómo citar este artículo:}

Pinilla, J. y Rialp, A. (2016). "Estrategia internacional, innovación y rendimiento: conglomerados estratégicos en pymes catalanas", Oikos No 41, 167-199, Escuela de Administración y Economía, Universidad Católica Silva Henríquez (UCSH), Santiago de Chile.

[http://ediciones.ucsh.cl/revistas.php]

Fecha de recepción: 30/03/2016

Fecha de aceptación: 17/05/2016 